\title{
Optimizing integrated imaging service delivery by tier in low-resource health systems
}

\author{
Kristen DeStigter ${ }^{1}$, Kara-Lee Pool ${ }^{*}$, Abimbola Leslie ${ }^{1}$, Sarwat Hussain ${ }^{3}$, Bien Soo Tan ${ }^{4}$, Lluis Donoso-Bach ${ }^{5}$ and
} Savvas Andronikou ${ }^{6}$

\begin{abstract}
Access to imaging diagnostics has been shown to result in accurate treatment, management, and optimal outcomes. Particularly in low-income and low-middle-income countries (LICS, LMICS), access is limited due to a lack of adequate resources. To achieve Sustainable Development Goal (SDG) 3, access to imaging services is critical at every tier of the health system. Optimizing imaging services in low-resource settings is best accomplished by prescriptive, integrated, and coordinated tiered service delivery that takes contextual factors into consideration. To our knowledge, this is the first recommendation for optimized, specific imaging care delivery by tier. A model for tier-based essential imaging services informs and guides policymakers as they set priorities and make budgetary decisions. In this paper, we recommend a framework for tiered imaging services essential to reduce the global burden of disease and attain universal health coverage (UHC). A lack of access to basic imaging services, even at the lowest tier of the health system, can no longer be justified by cost. Worldwide, affordable modalities of modern ultrasound and X-ray are becoming an accessible mainstay for the investigation of common conditions such as pregnancy, pneumonia, and fractures, and are safely performed and interpreted by qualified professionals. Finally, given the vast gap in access to imaging resources between LMICs and high-income countries (HICs), a scale-up of tiered imaging services in low-resource settings has the potential to reduce health disparities between, and within countries. As the access to appropriately integrated imaging services improves, UHC may be achieved.
\end{abstract}

Keywords: Global health, Radiology, Population health, Diagnostic imaging

\section{Key points}

- Access to imaging diagnostics has been shown to result in accurate treatment, management, and optimal outcomes.

- Access to imaging diagnostics in LMICs is limited due to lack of adequate resources.

- Tiered imaging services in low-resource settings have the potential to reduce health disparities between, and within countries, and may be implemented according to local context and setting.

\footnotetext{
*Correspondence: pool.kara.lee@gmail.com

2 RAD-AID International, 8004 Ellingson Drive, Chevy Chase, MD 20815,

USA

Full list of author information is available at the end of the article
}

- Investment in strengthening national policy around essential imaging diagnostics for tiered service delivery, with a focus on technology, human resources, infrastructure development, and quality management, will support primary care and specialty services for a healthy population.

\section{Introduction}

Imaging is an essential component of health systems where image-based diagnosis and intervention play a central role in individual patient care, through screening, diagnosis, treatment, and surveillance. Reliable and timely imaging results aid decision making for most health specialties and services, yet imaging services remain neglected or even invisible on the world stage. 
Lack of imaging diagnostics disproportionately impacts resource-limited areas that leaves millions of people without even basic reliable diagnostic imaging services. Sustainable Development Goals (SDGs) 3 has 13 targets to decrease the burden of disease, and even though imaging is an essential element of disease diagnosis, management, and eradication, none of the 26 key performance indicators mentions imaging [1-4]. The WHO Universal Health Coverage for the year 2030 (UHC 2030) promises continued expansion of primary care, surgical and obstetric services to the world's underserved regions, but says little about the imaging services required. The UHC 2030 jumps from preventive to curative, skipping the notion of "diagnosis" as a critical step [5]. The diagnostic gap for LICs and LMICs is more critical at the primary healthcare level, where access to even the simplest imaging is limited [6]. The health and economic benefits of investing in imaging services outweigh the cost, as calculated from future expenses avoided, with an estimated net survival benefit [4]. The disproportionately high burden of disease in low resource areas, especially sub-Saharan Africa [5], is worsened by a lack of access to affordable imaging technology, a limited skilled workforce, insufficient infrastructure, and limited processes for quality oversight, compounded by social inequities [6]. Many LMICs lack a placeholder for imaging services in their national health service strategic plans. A recent analysis of National Health Service Strategic Plans of all LICs and LMICs revealed that only $24 \%$ (19 countries) mentioned imaging/radiology services [7], which leaves imaging services unfunded, of low priority, and with little chance of operationalization.

In 2000, the UN Committee on Economic and Social Council (ECOSOC) described the right to the highest attainable standard of health, noting that health services should be available in adequate numbers, accessible, of good quality, and acceptable to all [8]. As a result of COVID-19, the UN Human Rights Council repeated that everyone, without exception, has the right to life-saving interventions [9]. Failure of the healthcare system to deliver services to low-income populations in LMICs has been attributed to lack of equity in health, demonstrating a need for fair distribution of health services, across all tiers of the healthcare system [10]. Tier-based imaging services addresses and promotes equity in access to basic imaging services.

The WHO Essential Diagnostic List (EDL) is a package of services in a pyramidal, tier-based healthcare system, where a primary health center serves the local community and refers to a higher level of care where expanded services are offered. This serves as a general recommendation for countries based on contextual circumstances, including the level of poverty, social preferences, operational challenges, and differences in disease burden [11]. To date, imaging has not been included in WHO EDLs across tiers [12].

In this paper, the top 21 diseases/conditions forecasted for 2030 (in the Global Burden of Disease Foresight data) [5] are converted to indications for imaging and matched with the radiological services required for primary diagnosis, diagnosis of complications, treatment, and surveillance (Table 1) based on the American College of Radiology (ACR) Appropriateness Criteria. Notably, imaging diagnosis and/or intervention is required across the board, underscoring the need to improve community access to imaging services through a tier-based system [13].

Many countries, including LICs and LMICs, have pyramidal-based health systems with tiers of service, with anything from 3-6 tiers depending on the country, e.g., Zambia has three tiers [14]. Lower tiers of health systems usually lack the skills, facilities, or both to manage some illnesses and may be compelled to refer patients to higher tiers that are better equipped with the resources needed for appropriate care [15]. Differentiation of the diagnostic imaging services into tiers organizes features of gradually increasing complexity in the availability of basic to advanced diagnostic technologies, personnel offering specialized services, and /or advanced therapeutic technologies that facilitate diagnosis and treatment of complex conditions [16]. Imaging services are well-suited to four tiers (Fig. 1) where modern basic X-ray and ultrasound imaging is adaptable for a primary healthcare level and the most advanced services are offered at the highest level.

The following are the necessary components of integrated tiered imaging service delivery: imaging technology, qualified human capacity, infrastructure (including information technology), and management of quality systems. In addition, an established and functional pattern for referrals is essential, with patients able to access higher levels of care as needed. Specific guidelines may not fit all countries where contextual considerations prevail.

The goal of this paper is to provide a framework for optimizing integrated imaging delivery services by tier in low-resource health systems. Imaging diagnosis is a collaborative activity that requires multiple elements that all are fit for purpose: technology, workforce, infrastructure, and quality management.

\section{Technology}

X-ray and ultrasound are considered traditional imaging modalities that yield the largest survival gains in low-income settings [17]. The WHO recommends that every woman should have at least one ultrasound during 
Table 1 Intersection of Forecasted (2030) Global Burden of Disease - top 21 causes - with Imaging Indication

\begin{tabular}{|c|c|c|c|c|c|}
\hline $\begin{array}{l}\text { Global burden disease foresight } 2030 \\
\text { list of \#1-21 causes of years of life } \\
\text { lost per } 100,000 \text { (both sexes, age- } \\
\text { standardized) [5] }\end{array}$ & $\begin{array}{l}\text { Screening } \\
\text { with } \\
\text { imaging }\end{array}$ & $\begin{array}{l}\text { Imaging for primary } \\
\text { diagnosis (directly or } \\
\text { contributory) }\end{array}$ & $\begin{array}{l}\text { Imaging for diagnosis of } \\
\text { complications of disease }\end{array}$ & $\begin{array}{l}\text { Imaging- } \\
\text { guided } \\
\text { treatment }\end{array}$ & $\begin{array}{l}\text { Imaging } \\
\text { surveillance }\end{array}$ \\
\hline 1. Cardiovascular Disease [13] & & $x$ & $x$ & $x$ & $x$ \\
\hline 2. Neoplasm $[12,19]$ & $x$ & $x$ & $x$ & $x$ & $x$ \\
\hline $\begin{array}{l}\text { 3. Diarrhea/lower respiratory tract infection } \\
\text { [13] }\end{array}$ & & $x$ & $x$ & & \\
\hline 4. Neonatal conditions [13] & & $x$ & $x$ & $x$ & $x$ \\
\hline 5. Chronic non-communicable diseases [13] & & $x$ & $x$ & $x$ & $x$ \\
\hline 6. HIV/AIDS/TB [13] & $x$ & $x$ & $x$ & $x$ & $x$ \\
\hline $\begin{array}{l}\text { 7. Unintentional injuries (falls, drowning, } \\
\text { fire) [13] }\end{array}$ & & $x$ & $x$ & $x$ & $x$ \\
\hline 8. Transport injuries [13] & & $x$ & $x$ & $x$ & $x$ \\
\hline 9. Chronic respiratory disease [13] & & $x$ & $x$ & $x$ & $x$ \\
\hline 10. Self-harm and violence [13] & & $x$ & $x$ & $x$ & \\
\hline $\begin{array}{l}\text { 11. Neglected tropical diseases (NTDs), } \\
\text { malaria }\end{array}$ & & $x$ & $x$ & $x$ & $x$ \\
\hline 12. Other non-communicable diseases [13] & & $x$ & $x$ & $x$ & $x$ \\
\hline 13. Neurological [13] & & $x$ & $x$ & $x$ & $x$ \\
\hline 14. Cirrhosis [13] & & $x$ & $x$ & $x$ & $x$ \\
\hline 15. Digestive diseases [13] & & $x$ & $x$ & $x$ & $x$ \\
\hline 16. Mental diseases [13] & & $x$ & $x$ & $x$ & $x$ \\
\hline 17. Other communicable (STDs) [13] & & & $x$ & & \\
\hline 18. Nutritional deficiencies & & & $x$ & $x$ & \\
\hline 19. War and disaster [13] & & $x$ & $x$ & $x$ & $x$ \\
\hline 20. Maternal disorders [13] & $x$ & $x$ & $x$ & $x$ & $x$ \\
\hline 21. Musculoskeletal [13] & & $x$ & $x$ & $\mathrm{x}$ & $x$ \\
\hline
\end{tabular}

pregnancy [14]. Advanced imaging modalities such as computed tomography scan (CT), magnetic resonance imaging (MRI), and positron emission tomography (PET) are now standard practice in the diagnosis of many diseases. New applications enabling telemedicine and artificial intelligence for disease detection and workflow efficiency will continue to change the care of patients in all tiers.

Constraints for achieving access to all modalities at all levels of the health system include high costs and lack of availability of equipment, infrastructure, and skilled human resources [15]. New equipment is expensive, often compounded by taxes and duties. Often, all the costs of ownership are not considered, including physical space, siting, licenses, network, and consumables. Installation failures due to inconsistent power sources, poor network connectivity, and lack of qualified personnel are common [18]. Many sales in LMICs use third-party distributors for new and refurbished equipment, limiting application training for personnel and resulting in lower confidence to use the equipment. Additionally, many donated devices lack warranties, installation support, user manuals, and even available parts. These challenges affect technology ownership at all tiers of imaging service delivery but are least able to be addressed at the lowest tiers due to the requirement for skilled human resources. Often, radiologists and other imaging specialists are not consulted when imaging equipment is purchased even though adequate, and appropriate imaging technologies fit for purpose, are what empowers healthcare teams to provide optimal patient care. In addition, competing priorities become limiting factors to technology procurement, especially in LICs and LMICs.

In the integrated, tiered healthcare system the various imaging technology needs for each tier are determined based on imaging testing needs, resource constraints, population needs, and infrastructure requirements [16].

For tier 1 in a limited resource environment, point-ofcare ultrasound (POCUS) and X-ray should be available for reducing both underdiagnosis and overdiagnosis [17, 19] (Figs. 2 and 3). Basic ultrasound for obstetric care, for example, is a primary care requirement in Uganda [20]. POCUS requires no additional skilled personnel, requires a minimal power supply, is rugged for remote 


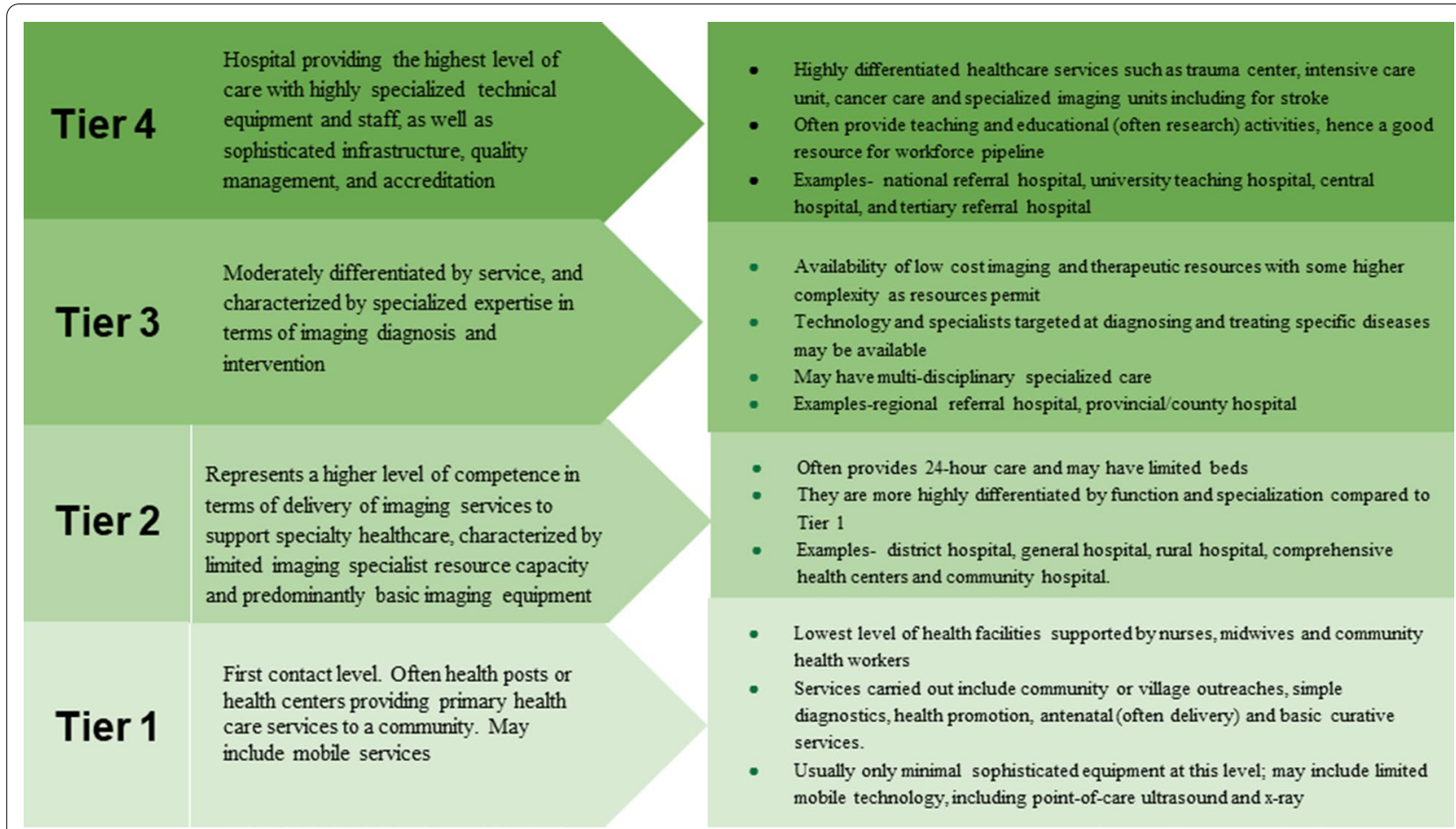

Fig. 1 Tiers and levels of Health Systems. A darker color denotes a higher level of care with higher complexity and requirement for more specialized human and technological resources

settings, and is relatively easy to teach and learn. It requires less infrastructure and training than other imaging modalities such as CT scans or MRIs. POCUS in a low-resource setting in rural Tanzania showed a change in diagnostic impression in over 40 percent of cases and helped providers identify new or additional diagnoses, improving patient care and outcomes [21]. Rapid point-of-care (POC) diagnostics are recommended by the WHO because they achieve the greatest impact on disease prevalence by improving the quality of care for screening programs and for individuals [22].

Tier 2 level facilities are usually the first level of referral and at the minimum should have comprehensive ultrasound plus X-ray and can include CT, MRI, fluoroscopy (FL), basic interventional radiology (B-IR), or even mammography, if resources (qualified staff and quality management) allow. Since clinical services offered at Tier 3 are more differentiated in terms of function and expertize, CT, MRI, FL, basic interventional radiology, and mammography services should be available at the minimum, and where more resources are available, complex interventional radiology with subspecialized protocols, advanced breast imaging (ABI), and PET could be added.

Tier 4 levels are the highest-level referral centers, often national referral and academic hospitals, that are tasked with providing the highest level of healthcare including complex IR, subspecialized IR (e.g., interventional neurology), ABI, PET, nuclear medicine (NM) and imageguided or image-directed therapies should be available, over and above Tier 3 services.

Availability of essential technology matching the most common indications for imaging at each tier can provide cost-effective measures through improved screening, diagnosis, treatment, and outcomes. For example, the scaling up of imaging services simulated for eleven cancer types has been shown to avert about 2.46 million deaths, accounting for $3.2 \%$ of worldwide deaths and 54.92 million life-years saved [4]. As a further example, Interventional Radiology (IR) could provide greater benefits for LICs and LMICs, relative to HICs, due to the high mortality rates associated with the surgical and anesthesia complications they experience in practice [23].

\section{Human resources}

Human resources at each tier should be appropriate for the services provided. Radiologists being qualified medical doctors with additional specialty training in diagnostic imaging represent the highest tier of the diagnostic imaging workforce. Limiting factors for producing radiologists locally include the availability of medical graduates, infrastructure (equipment), and 


\title{
Imaging Technology Strategy by Health System Tier
}

\author{
Technology Resource Need
}

Where care is delivered

Complex IR, Subspecialized IR,

ABI, PET, NM, Therapies

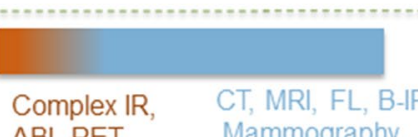

ABI, PET

Mammography
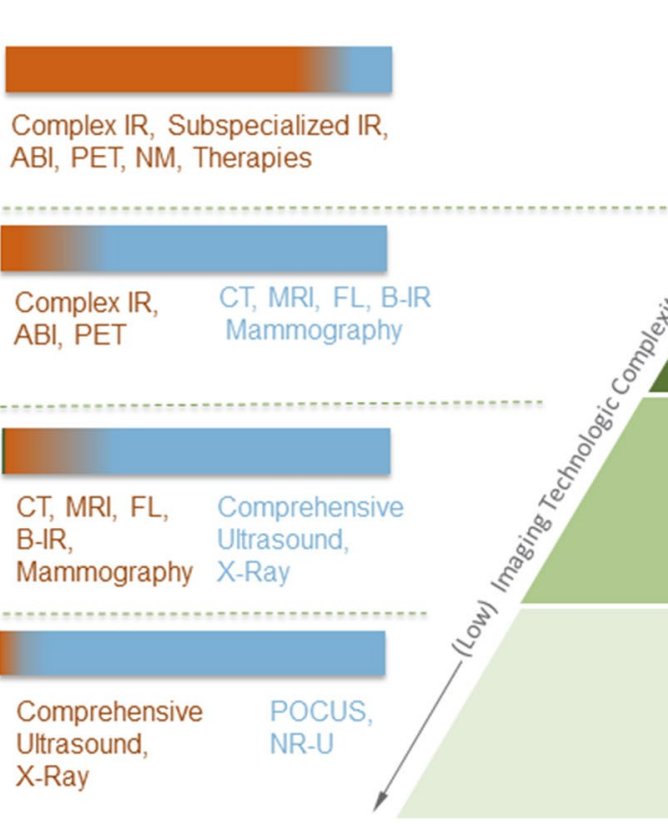

Tier 2

Intermediate Cost
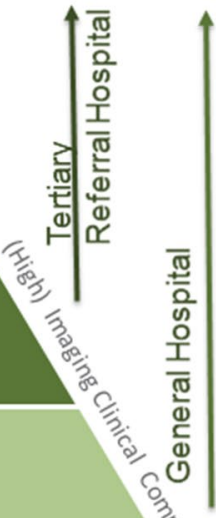

Fig.

Fig. 2 Recommended imaging technology strategy by health system tier. Higher levels on the pyramid denote increasing complexity. For each tier, red indicates a requirement for higher complexity of technology while blue indicates lower complexity of technology. (POCUS = Point of Care ultrasound; $\mathrm{NR}-\mathrm{U}=$ non-radiologist ultrasound; $\mathrm{CT}=$ computed tomography; $\mathrm{MRI}=$ magnetic resonance imaging; $F L=$ fluoroscopy; $\mathrm{B}-\mathrm{IR}=\mathrm{BASIC}$ Interventional Radiology; $\mathrm{ABI}=$ advanced breast imaging; $\mathrm{PET}=$ positron emission tomography; $\mathrm{NM}=$ nuclear medicine; subspecialized $\mathrm{IR}=$ neurointerventional)

expertize (qualified radiologists) to provide training, usually in a four-to-five-year program.

General radiologists, accredited nationally as medical specialists, provide services in most fields of diagnostic imaging and some basic interventional radiology. They interpret diagnostic imaging with physician-level insight and should be available in tiers 2-4 where specialized radiologic equipment is available (Fig. 4). Radiologists can become subspecialized with further training, in areas such as neuroradiology, cardiothoracic imaging, abdominal imaging, breast imaging, pediatric imaging, musculoskeletal imaging, and vascular interventional radiology. Subspecialist radiologists are ideally suited for tier 4, a tertiary referral, and academic centers, but are a scarce resource in LICs and LMICs. Although not required in tier 1, general radiologists can play a role in performing primary-care level ultrasound and training POCUS practitioners.

Non-radiologists, including radiographers (X-ray technologists), sonographers, physicists, nurses, etc., are integral members of diagnostic imaging services and are required at all 4 tiers to provide the images. Formal training on modality applications is necessary for accreditation as a technologist/ sonographer, followed by regular in-service training and performance evaluation.

Established radiologist and technologist training programs exist in many LICs and LMICs but some countries lacking full radiologist training capabilities, send candidates to neighboring and distant countries for training.

Ideally, a sonographer (technologist) should staff tier 1 but imaging services can be offered (performed and interpreted) by training a non-radiologist/ non-technologist to perform some imaging through 'task shifting, a 


\section{Ultrasound Imaging Strategy by Health System Tier}

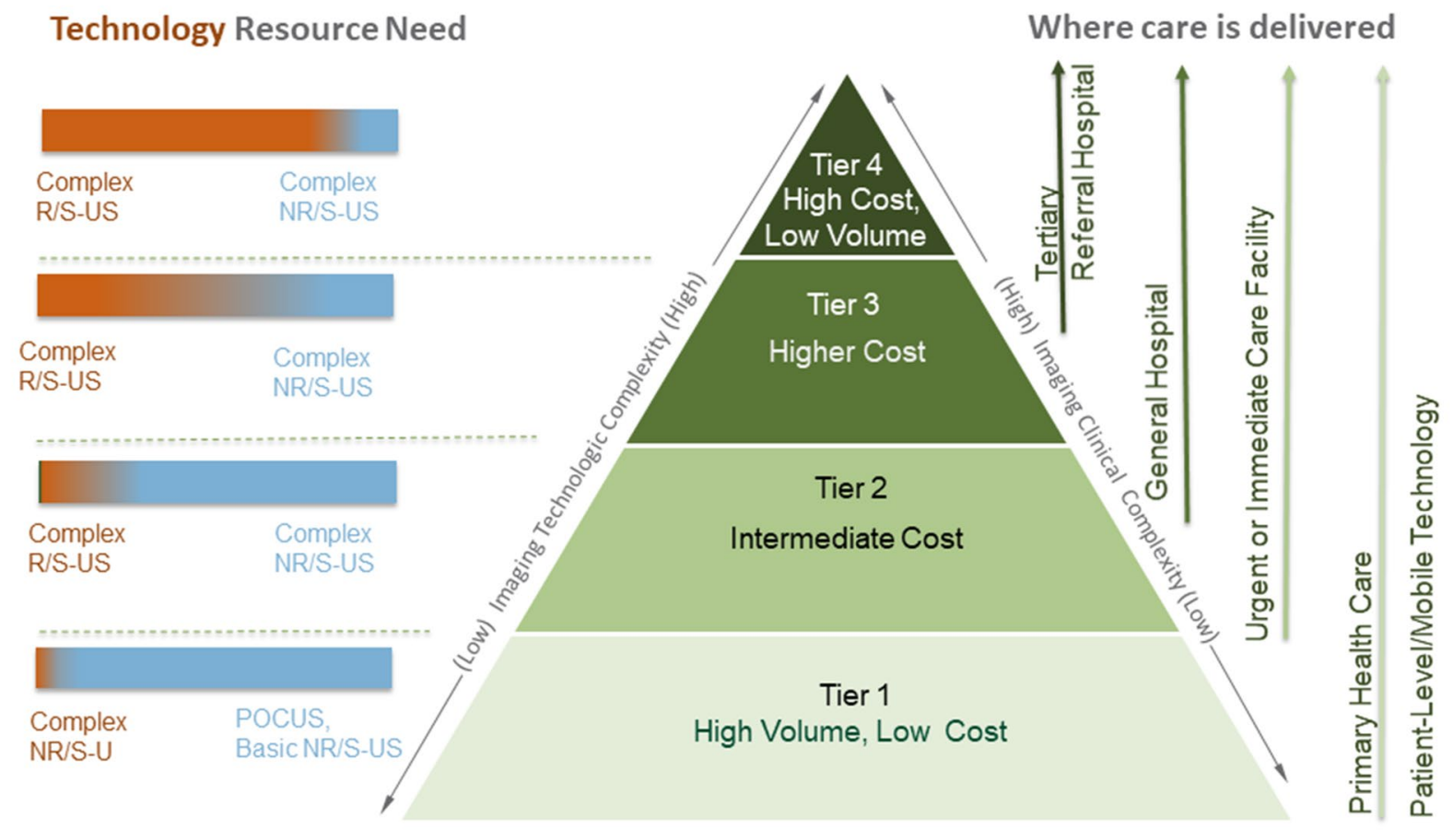

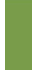

Fig. 3 Recommended ultrasound imaging strategy by health system tier. Higher levels on the pyramid denote increasing complexity. For each tier, red indicates a requirement for higher specialization of service (technology and personnel), while blue indicates lower specialization requirements (POCUS = Point of Care ultrasound; NR/S-US= non-radiologist/non-specialist ultrasound; complex R/S-US=radiologist/specialist ultrasound)

strategy for addressing skilled healthcare worker shortages in developing countries [24, 25]. Task shifting is prevalent in African tier 1 facilities in Malawi, Uganda, and Zimbabwe where the benefits such as reduced waiting, faster turnaround times, and lower costs outweigh possible harms [25-29]. A global shortage of radiologists and increased demand for image interpretation has resulted in task-shifting to radiographers [30]. Primarily this relates to reporting appendicular X-rays from emergency departments [31] with evidence that radiographers are as accurate as radiologists [31-33] and more accurate than medical officers [34]. Implementation of teleradiology services and/or Artificial Intelligence (AI) can augment task shifting in tier 1 facilities. Radiographer extenders/advanced practice providers are well established in the United Kingdom [35-38] at all tiers of service but do not replace radiologists in tiers 2-4.

One notable form of task-shifting is the performance of point-of-care ultrasound (POCUS) by non-radiologists (physicians and non-physicians). POCUS has many applications, both in emergency/specialty tier 2-4 settings and non-emergency primary care settings (tier 1). In Uganda, rural non-physician clinicians use POCUS more often than imaging department-performed studies [39]. Task-shifting ultrasound of the breast is an appropriate alternative to mammography in Africa, considering the lack of infrastructure for mammography programs and the natural history of the disease locally [40]. POCUS for HIV and TB diagnosis is also well established [41].

Health systems should optimize recruitment and retention of qualified staff, especially those with advanced training and skills. On the other end of the tier-based system, barriers to task shifting include concerns about funding, licensing, and health worker concerns [24, 42]. Task-shifting also requires resources, training, and innovative strategies to be successful, and safe and its implementation must be contextual and accompanied by supervision and quality management $[20,43,44]$. 


\section{Imaging Workforce/Human Resource Strategy by Health System Tier}

Human Resource Need

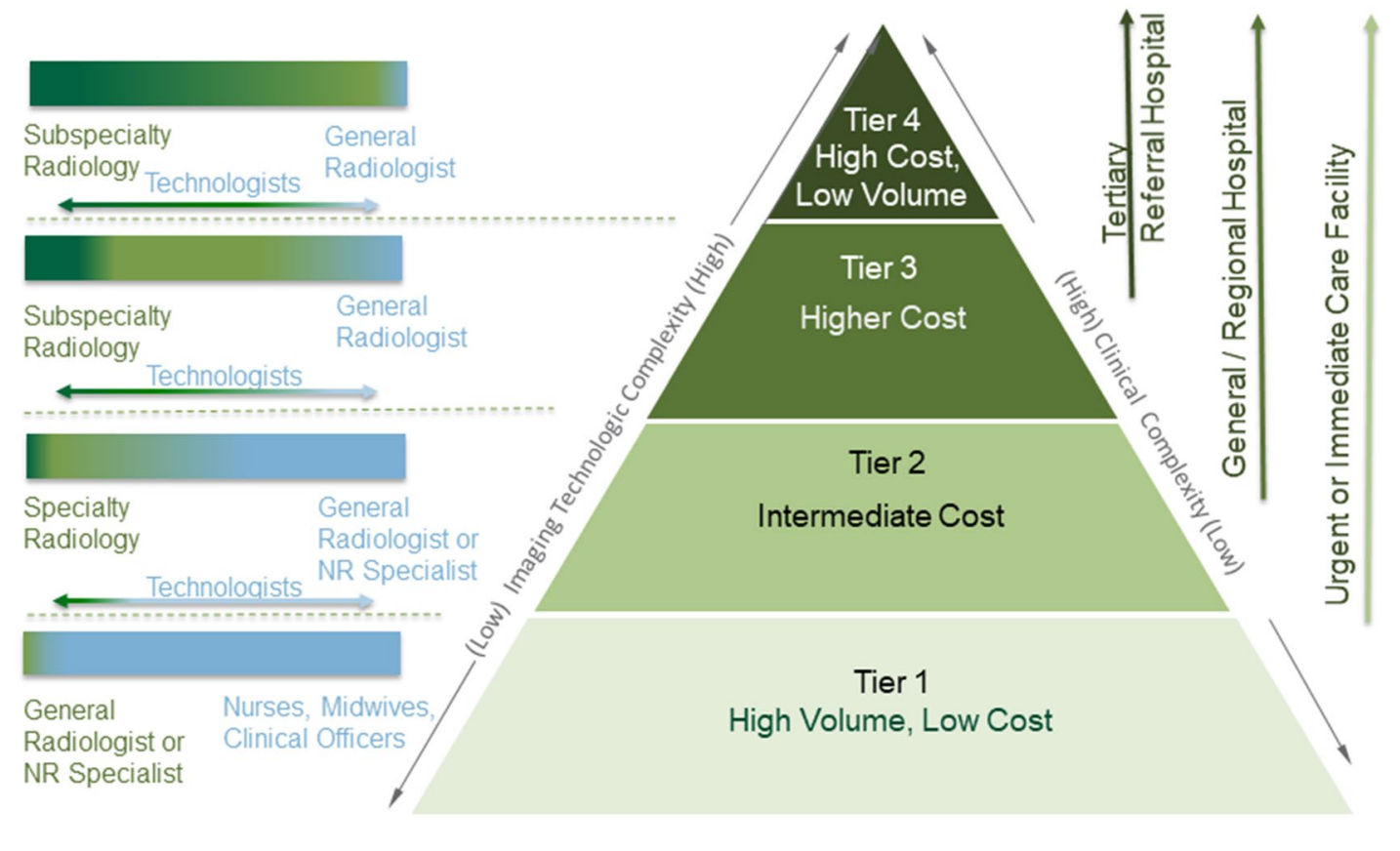

Where care is delivered

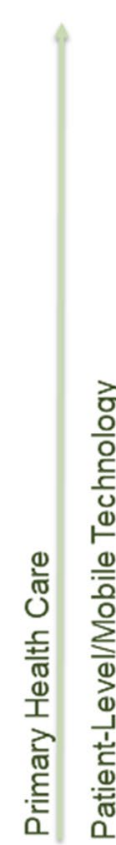

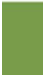

Fig. 4 Recommended imaging workforce/human resource strategy by health system tier. Higher levels on the pyramid denote increasing complexity and requirement for specialization of staff. Green denotes a requirement for higher-level specialization or even sub-specialization (e.g., neurointerventional radiology, oncologic imaging), while blue denotes lower-level specialization. Technologists / radiographers are required at all tiers above primary care centers and may also have a role and primary care level even though not essential (NR=Non-Radiology; technologists $=$ radiographic technologists, radiographers, sonographers)

\section{Infrastructure}

Infrastructure includes a wide range of complex building blocks ranging from physical structural components to supporting services like transportation, energy, and computer bandwidth. Unlike other aspects of health care, access to reliable infrastructure for even the most basic imaging services is vital to sustainability and function. The two most rudimentary infrastructure components required for tier 1 basic imaging services include electricity and internet access-both of which are priorities included in UN SDGs [45]. Many hospitals and clinics in LMICs do not even have access to reliable electricity sources whereas the world bank states that broadband internet access is "not a luxury, but a basic necessity for economic and human development" [46].

As the tiers increase in complexity of service, the infrastructure needs to increase significantly (Fig. 5). Building upon the basic energy source and internet integrity, several key infrastructure components are required to adequately sustain diagnostic ultrasound machines and X-ray units spanning tiers $1-4$. These include radiation safety with structural shielding, imaging consumables, climate control and maintenance. If tele-consults or artificial intelligence (AI) platforms are being implemented as an imaging service for tier 1 or higher, a more robust IT system will need to be in place, including a picture archiving and communication system (PACS). Each machine will have an endof-life (EOL) and end-of-service/support life (EOSL), which are both important to consider when procuring new or used products in order to ensure the optimal life of each machine. It is well known that donated imaging equipment and equipment procured without service contracts create challenges for LMICs and lead to inadequate equipment performance and use [18]. For referral healthcare centers in tiers $2-4$ that 


\section{Strategy for Imaging Infrastructure Resources by Health System Tier}

\section{Infrastructure Resource Need}

NM Lab

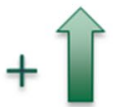

MRI Safety, EMR, Pharmacy, PALM services

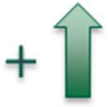

Structural, Climate Control, IT, Radiology Consumables,

Maintenance; Radiation Safety,

PACS, Communications

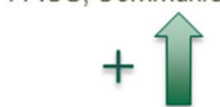

Energy Source, Internet Integrity

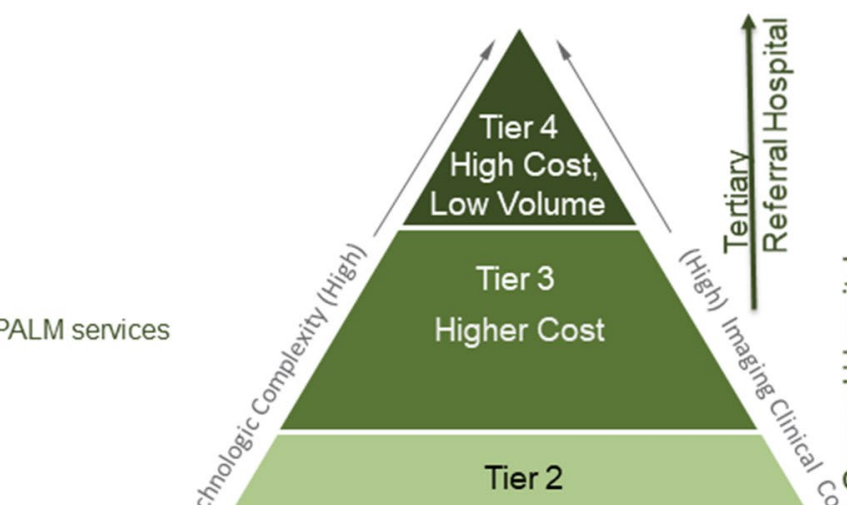

Intermediate Cost

Where care is delivered
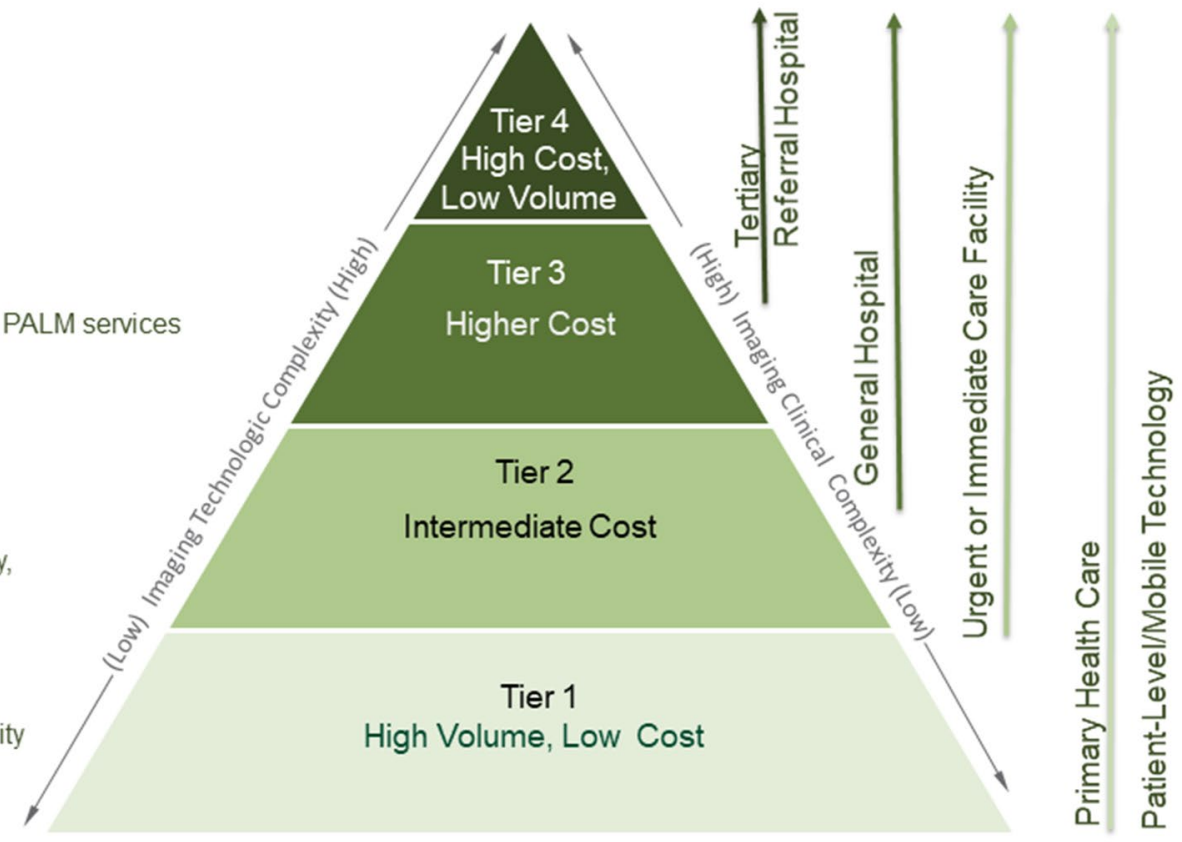

Fig. 5 Recommended imaging infrastructure resource strategy by health system tier. Higher levels on the pyramid denote increasing complexity and requirement for more sophisticated and specialized infrastructure. Green arrows indicate added infrastructure required at the next highest tier (POCT = Point-of-care testing; IT = Information Technology; PACS = Picture Archiving and Communication system; MRI= Magnetic resonance imaging; EMR = electronic medical record; $\mathrm{NM}=$ nuclear medicine; $\mathrm{PALM}=$ pathology and laboratory medicine)

provide multidisciplinary care, PACS, electronic medical records (EMR), and robust internet are essential.

Having insufficient access to transportation is a wellknown rate-limiting step to providing many social and economic opportunities including the ability to procure many advanced medical devices, the maintenance of these devices, the procurement of imaging consumables and medications. To sustain the complex imaging devices requires easy access roads, nearby airplane landing strips, or boat docks given the importance of maintenance of these machines.

It is important to note, that as technology evolves, the requirements of infrastructure will also evolve. For example, ultrasound, X-ray machines, CT, and even MRI devices are becoming more portable and require less space and power, yet the ability to store images and provide remote quality care through cloud-based telecommunication systems is becoming standard in many parts of the world. Therefore, infrastructure needs will require continued updates and the capacity to evolve with new innovation.

\section{Quality and oversight}

Unlike high-income countries (HICs), quality improvement programs are rarely legally mandated in LMICs. There is little published literature on imaging quality in LMICs, the majority focusing on quality control or radiation protection [46-48]. Very few papers report on quality improvement (QI) programs [47]. A survey of 23 leading hospitals in Ghana revealed complete absence of imaging quality assurance or quality improvement plans across the polled hospitals [49].

All LMICs can implement a quality improvement program. The QI vision should begin with the belief by political and healthcare leadership, that all people have a right to quality care so that this can translate into national healthcare quality improvement initiatives of diagnostic imaging services. A parallel step is to enact healthcare 
quality legislation featuring diagnostic services, and allimportant buy-in from the stakeholders.

QI plans can be customized [50] by leadership and staff to fit all 4 Tiers of service (Fig. 6). Key elements of a QI plan are system orientation, and patient-centeredness, team building, education and training, data collection and analysis, infection control (especially hand hygiene), process checklists, diagnostic protocols, verification of the processes, and feedback. Data must be reported to the QI committee and up the chain of command, and QI plans should be revised according to lessons learned.

A QI plan for a tier 1 provided by non-radiologists performed ultrasound requires staff training and skills verification, Quality Control (QC) of acquired images, staying within accreditation standards, continuous skills enhancement, retention initiatives, and QI plan refinement, based on staff and patient feedback. For LMICs, the absence of skills verification is probably the weakest link in QI.
The focus of QI in Tier 2 is similar to Tier 1, but the presence of onsite radiologists allows for on-site supervision, real-time image $\mathrm{QC}$, process verification, and data review, with real-time feedback to staff.

QI is pivotal to Tier 3, and critical for Tier 4 because these incorporate a full range of imaging modalities and procedures. Services, such as neuro-intervention and endovascular are highly risky, therefore skills verification, credentialing, and compliance with processes and protocols must be verified and recorded, for review. Departmental QI committees report to a quality officer, usually a senior radiologist. The committee analyzes QI data, especially complications and near misses, using statistical tools (e.g., Pareto chart, fish-bone diagram, or control chart). A commonly used methodology for affecting change is the Plan-Do-Study-Act (PDSA) cycle consisting of planning a change, trying it out on a small scale, observing the results, and acting on what is learned. All QI systems must adopt a methodology for change.

\section{Imaging Quality Management \& Oversight Strategy by Health System Tier}

\section{Quality Oversight Resource Need}

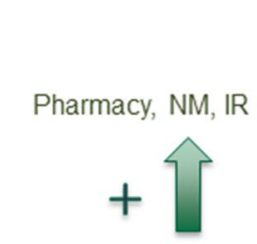

MRI Safety, Accreditation

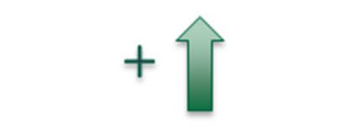

Radiation Safety, National Oversight

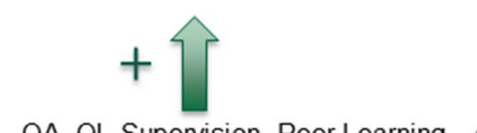

QA, QI, Supervision, Peer Learning, M\&E, outcomes tracking

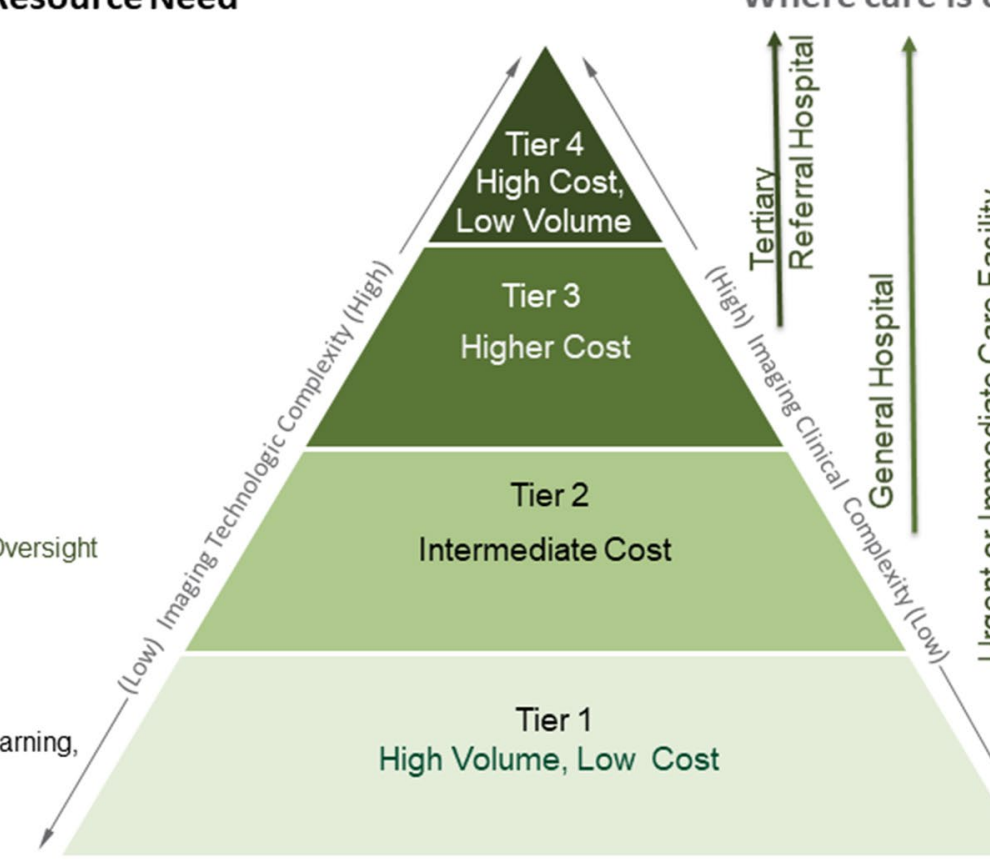

\section{Where care is delivered}

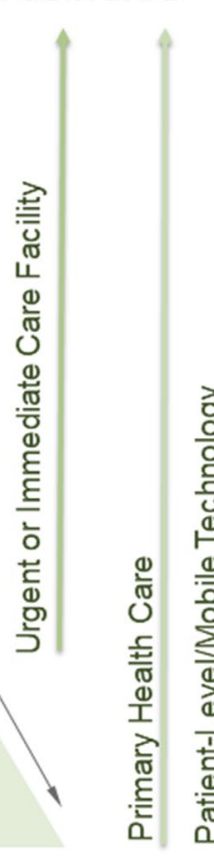


Speaking up with ideas, and asking questions is to be encouraged at all tiers of imaging service.

\section{Conclusion}

Strengthening national policy around tiered diagnostic imaging will support primary care and specialty services for the overall improved health of the population. Investment should be made into service delivery, technology, capacity, and capability strengthening of human resources, infrastructure development, and quality management systems. We have provided a novel framework for a tiered diagnostic imaging service, flexible enough to account for available resources in different contexts and settings. The COVID-19 pandemic proved that having a fluid, yet well-defined framework allows for maximum capacity utilization, even when the system is strained [51, 52]. In applying this framework, local radiologists should be empowered to inform decisions, support national policies, norms, and standards, and participate in cost-effectiveness studies that guide future policy.

\begin{abstract}
Abbreviations
ABI: Advanced breast imaging; B-IR: Basic interventional radiology; Complex IR Complex interventional radiology; Complex R/S-US: Complex radiologist/specialist ultrasound; CT: Computed tomography; EOL: End-of-life (equipment); EOSL: End-of service/support life (equipment); FL: Fluoroscopy; MRI: Magnetic resonance imaging; NM: Nuclear medicine; NR/S-US: Non-radiologist/nonspecialist ultrasound; NR: Non-radiologist; NR-U: Non-radiologist ultrasound; PACS: Picture archiving and communication system; PET: Positron emission tomography; POCUS: Point of care ultrasound; QC: Quality control; QI: Quality improvement; Subspecialized-IR: Subspecialized interventional radiology.
\end{abstract}

\section{Authors' contributions}

All authors contributed to conception, design, analysis, data acquisition, writing and editing this manuscript. All authors read and approved the final manuscript.

\section{Funding}

No funding is associated with this manuscript.

\section{Availability of data and materials}

Not applicable.

\section{Declarations}

Ethics approval and consent to participate

Not applicable. No IRB was required for this manuscript.

\section{Consent for publication}

Not applicable.

\section{Competing interests}

There are no competing interests for any authors.

\section{Author details}

${ }^{1}$ Department of Radiology, Larner College of Medicine, University of Vermont, 111 Colchester Avenue Main Campus, McClure, Level 1, Burlington, VT 05401 USA. ${ }^{2}$ RAD-AID International, 8004 Ellingson Drive, Chevy Chase, MD 20815, USA. ${ }^{3}$ Department of Radiology, University of Massachusetts, 55 North Lake Ave, Worcester, MA 01655, USA. ${ }^{4}$ Department of Vascular and Interventional Radiology, Singapore General Hospital, Outram Rd, Singapore 169608, Singapore. ${ }^{5}$ Department of Medical Imaging, Hospital Clínic of Barcelona, University of Barcelona, C. de Villarroel, 170, 08036 Barcelona, Spain. ${ }^{6}$ Department of Pediatric Radiology, The Children's Hospital of Philadelphia and the Perelman School of Medicine, University of Pennsylvania, Philadelphia, USA.

Received: 12 May 2021 Accepted: 11 Auqust 2021

Published online: 16 September 2021

\section{References}

1. dpicampaigns. Take action for the sustainable development goals. Retrieved from https://www.un.org/sustainabledevelopment/susta inable-development-goals/

2. Universal Health Coverage Report. Retrieved from https://www.who.int/ healthinfo/universal_health_coverage/report/uhc_report_2019.pdf

3. Wilson ML, Atun R, DeStigter K et al (2019) The lancet commission on diagnostics transforming access to diagnostics. Lancet. https://doi.org/ 10.1016/S0140-6736(19)31052-9

4. Hricak MM, Abdel-Wahab M, Atun R et al (2021) Medical imaging and nuclear medicine: a lancet oncology commission. Lancet Oncol. https:// doi.org/10.1016/S1470-2045(20)30751-8

5. GBD Foresight. http://vizhub.healthdata.org/gbd-foresight/forecasting

6. Balogh EP, Miller BT, Ball JR (2015) Improving diagnosis in health care. https://doi.org/10.17226/21794

7. Shah D, DeStigter K, van den Broek-Altenburg E, Horton S, Dahn B (2021) The inclusion of laboratory and imaging diagnostics in national health service strategic plans: an analysis of 79 low income and lower-middleincome countries

8. OHCHR International Covenant on Economic, Social and Cultural Rights. Retrieved from https://www.ohchr.org/EN/Professionallnterest/Pages/ CESCR.aspx

9. OHCHR. Retrieved from https://www.ohchr.org/en/NewsEvents/Pages/ DisplayNews.aspx? News $\mid \mathrm{D}=25746 \&$ Lang $\mid \mathrm{D}=\mathrm{E}$

10. Health equity: challenges in low-income countries. Afr Health Sci 9:S49 (2009)

11. Second WHO model list of essential in vitro diagnostics. https://www. who.int/medical_devices/publications/Standalone_document_v8.pdf? $\mathrm{ua}=1$

12. World Health Organization: Regional office for Europe. World cancer report: cancer research for cancer development (2020)

13. American college of radiology-appropriateness criteria. https://acsearch. acr.org/list?_ga=2.136760880.1802011192.1616357807-1193651604. 1616357807

14. Mbewe C, Chanda-Kapata P, Sunkutu-Sichizya V et al (2020) An audit of licensed Zambian diagnostic imaging equipment and personnel. Pan Afr Med J 36

15. Hensher M, Price M, Adomakoh S (2006) Referral hospitals. In: Disease control priorities in developing countries, 2nd edn. The International Bank for Reconstruction and Development/The World Bank

16. WHO levels of health services. https://2018.iupesm.org/wp-content/ uploads/2014/06/WHO-LevelsofHealthServices.pdf

17. Ward ZJ, Scott AM, Hricak $\mathrm{H}$ et al (2020) Estimating the impact of treatment and imaging modalities on 5-year net survival of 11 cancers in 200 countries: a simulation-based analysis. Lancet Oncol 21:1077-1088

18. Equipment in the global radiology environment: why we fail, how we could succeed. https://escholarship.umassmed.edu/cgi/viewcontent.cgi? article $=1079 \&$ context $=$ jgr

19. World Health Organization. Report on cancer setting priorities, investing wisely and providing care for all (2020). PDF

20. Service standards and service delivery standards for the health sector. http://library.health.go.ug/publications/quality-assurance-quality-impro vement/service-standards-and-service-delivery-standards

21. Reynolds TA, Amato S, Kulola I, Chen CJ, Mfinanga J, Sawe HR (2018) Impact of point-of-care ultrasound on clinical decision-making at an urban emergency department in Tanzania. PLoS One 13

22. World Health Organization (2017) A guide to aid the selection of diagnostic tests. Retrieved from https://www.who.int/bulletin/volumes/95/9/ 16-187468/en/

23. Yang Y, Moore A, Gaupp FL, Ahuja R, Sanyika C, Makris GC (2021) A call to action; an open letter to WHO from the international interventional 
radiology community. CVIR Endovasc 4:3. https://doi.org/10.1186/ s42155-020-00195-2

24. Mpunga T, Hedt-Gauthier BL, Tapela N et al (2016) Implementation and validation of telepathology triage at cancer referral center in rural Rwanda. J Glob Oncol 2:76-82

25. Nkhata MJ, Muzambi M, Ford D et al (2016) Shifting human resources for health in the context of ART provision: qualitative and quantitative findings from the Lablite baseline study. BMC Health Serv Res 16:660

26. Vojnov L, Taegtmeyer M, Boeke C et al (2019) Performance of non-laboratory staff for diagnostic testing and specimen collection in HIV programs: a systematic review and meta-analysis. PLoS One 14

27. Nightingale J, Hogg P (2007) The role of the Gl radiographer: a United Kingdom perspective. Radiol Technol 78:284-290

28. Feiring $E$, Lie $A E$ (2018) Factors perceived to influence implementation of task shifting in highly specialised healthcare: a theory-based qualitative approach. BMC Health Serv Res 18:899

29. Bautista-Arredondo S, Sosa-Rubi SG, Opuni M et al (2018) Influence of supply-side factors on voluntary medical male circumcision costs in Kenya, Rwanda, South Africa, and Zambia. PLoS One 13:e0203121

30. Wuni A-R, Courtier N, Kelly D (2020) Opportunities for radiographer reporting in Ghana and the potential for improved patient care. Radiography 26:e120-e125

31. McLaughlin L, McConnell J, McFadden S, Bond R, Hughes C (2017) Methods employed for chest radiograph interpretation education for radiographers: a systematic review of the literature. Radiography 23:350-357

32. Brealey S, Scally A, Hahn S, Thomas N, Godfrey C, Coomarasamy A (2005) Accuracy of radiographer plain radiograph reporting in clinical practice: a meta-analysis. Clin Radiol 60

33. Murphy A, Ekpo E, Steffens T, Neep MJ (2019) Radiographic image interpretation by Australian radiographers: a systematic review. J Med Radiat Sci 66:269-283

34. du Plessis J, Pitcher R (2015) Towards task shifting? A comparison of the accuracy of acute trauma-radiograph reporting by medical officers and senior radiographers in an African hospital. Pan Afr Med J 21

35. Hardy M, Johnson L, Sharples R, Boynes S, Irving D (2016) Does radiography advanced practice improve patient outcomes and health service quality? A systematic review. Br J Radiol 89

36. Antwi WK, Akudjedu TN, Botwe BO (2021) Artificial intelligence in medical imaging practice in Africa: a qualitative content analysis study of radiographers' perspectives. Insights Imaging 12(1):80

37. Botwe BO, Akudjedu TN, Antwi WK (2021) The integration of artificial intelligence in medical imaging practice: perspectives of African radiographers. Radiography 20:S1078-8174

38. Botwe B, Antwi WK, Arkoh S, Akudjedu TN (2021) Radiographers perspectives on the emerging integration of artificial intelligence into diagnostic imaging: the Ghana study. J Med Radiat Sci. https://doi.org/10.1002/jmrs. 460
39. Stolz LA, Hilary K, Dreifus B et al (2014) Point-of-care ultrasound training for non-physician emergency care practitioners in rural Uganda. Lancet. https://doi.org/10.1016/S2214-109X(15)70060-4

40. Galukande M, Kiguli-Malwadde E (2010) Rethinking breast cancer screening strategies in resource-limited settings. Afr Health Sci 10:89-92

41. Ruby LC, Kadavigere R, Sheshadri S, Saravu K, Bélard S (2021) Pulmonary aspergilloma on transthoracic ultrasound. Infection. https://doi.org/10. 1007/s15010-021-01589-7

42. Liu R, Huang L, Yang Q et al (2018) Investigation on task shifting of HIV/ AIDS follow-up management workers in new launched areas, China. Int J Environ Res Public Health 15

43. Semakula-Katende NS, Andronikou S, Lucas S (2016) Digital platform for improving non-radiologists' and radiologists' interpretation of chest radiographs for suspected tuberculosis - a method for supporting taskshifting in developing countries. Pediatr Radiol 46:1384-1391

44. Woznitza N, Piper K, Burke S, Bothamley G (2018) Chest X-ray interpretation by radiographers is not inferior to radiologists: a multireader, multicase comparison using JAFROC (Jack-knife alternative free-response receiver operating characteristics) analysis. Acad Radiol 25:1556-1563

45. Overview. https://www.worldbank.org/en/topic/transport/overview

46. National Council on Radiation Protection and Measurements \& NCRP (1988) Quality assurance for diagnostic imaging equipment: recommendations of the national council on radiation protection and measurements. National Council on Radiation

47. Patel V, Sindhwani G, Gupta M et al (2017) A comprehensive approach towards quality and safety in diagnostic imaging services: our experience at a rural tertiary health care center. J Clin Diagn Res 11:TC10

48. Taylor MJ, McNicholas C, Nicolay C, Darzi A, Bell D, Reed JE (2014) Systematic review of the application of the plan-do-study-act method to improve quality in healthcare. BMJ Qual Saf 23:290-298

49. Patients' satisfaction with diagnostic radiology services in two major public and private hospitals in Ghana. Int J Radiol Radiat Therapy 1:62-65 (2016)

50. Kruskal JB, Eisenberg R, Sosna J, Yam CS, Kruskal JD, Boiselle PM (2011) Quality initiatives: Quality improvement in radiology: basic principles and tools required to achieve success. Radiographics 31(6):1499-1509

51. Elshami W, Akudjedu TN, Abuzaid M et al (2021) The radiology workforce's response to the COVID-19 pandemic in the Middle East, North Africa and India. Radiography 27(2):360-368

52. Mendel JB (2020) COVID-19 pandemic and radiology: facts, resources, and suggestions for near-term protocols. J Glob Radiol 6(1):1100

\section{Publisher's Note}

Springer Nature remains neutral with regard to jurisdictional claims in published maps and institutional affiliations.

\section{Submit your manuscript to a SpringerOpen ${ }^{\circ}$ journal and benefit from:}

- Convenient online submission

- Rigorous peer review

- Open access: articles freely available online

- High visibility within the field

- Retaining the copyright to your article

Submit your next manuscript at $\boldsymbol{\nabla}$ springeropen.com 